\title{
Simulación de una Cadena de Abastecimiento
}

\author{
Ma. de Lujan Betria, Luis P. Lara \\ Departamento de Física, Facultad de Ciencias Exactas, Ingeniería y Agrimensura, \\ Universidad Nacional de Rosario, Av. Pellegrini 250 (S2000BPT) Rosario, Argentina \\ lujanbetria@hotmail.com; lplara@fceia.unr.edu.ar
}

\begin{abstract}
El objetivo de este trabajo es el estudio del comportamiento dinámico del nivel de inventarios en una cadena de abastecimiento con recolección de los ítems utilizados. A través de la Dinámica industrial, se propone un modelo matemático conformado por ecuaciones diferenciales que relacionan los niveles de inventarios y los flujos entre ellos. El modelo constituye una herramienta de simulación experimental que puede ser utilizada bajo diferentes condiciones del sistema.
\end{abstract}

Palabras Clave: Dinámica industrial, Simulación, Cadena de abastecimiento.

\section{Introducción}

Este trabajo presenta el desarrollo de un modelo matemático de una cadena de abastecimiento cerrada. El modelo incluye el canal tradicional constituido por la fábrica, un distribuidor y los clientes; y el canal reverso [5,10] que incluye las actividades de recolección, inspección y remanufactura de los ítems utilizados. Los productos recolectados vuelven a la fábrica a través de este canal reverso. Se considera un único producto. El modelo no considera el reaprovisionamiento de materia prima.

Estas características hacen al modelo especialmente aplicable a casos en que la materia prima constituye un recurso escaso, ya sea por ser no renovable o por cualquier otro factor limitante. El mismo se podría aplicar en la industria de la madera a la fabricación de muebles, en la industria del aluminio a la fabricación de ollas, en la industria del cobre a la fabricación de cables. De hecho, actualmente, diversas empresas de los rubros mencionados alientan a los consumidores, a través de políticas promocionales a canjear su producto usado por uno nuevo. El producto usado se da en parte de pago y la empresa lo remanufactura para su posterior venta.

El objetivo general de este trabajo es analizar el comportamiento dinámico del sistema [3]. El objetivo especifico, es estudiar el impacto del canal reverso sobre el canal tradicional, en particular, sobre el comportamiento de los inventarios y sobre el tiempo de agotamiento de la materia prima.

Si bien, el comportamiento de los inventarios, constituye un fenómeno discreto, la descripción del sistema se realizó considerando que los cambios ocurren continuamente.

El modelo constituye una herramienta de simulación experimental, que puede ser utilizada para analizar el comportamiento del sistema bajo diferentes condiciones [9]. 
La metodología utilizada es la Dinámica Industrial. La dinámica industrial es una metodología de análisis del comportamiento en el tiempo de sistemas organizacionales desarrollada por Jay W. Forrester [6,7] y sus colaboradores en el MIT en la década del 60. Su objetivo es la comprensión y el mejoramiento del desempeño de los sistemas bajo estudio. Esta metodología considera a la empresa como un todo con el fin de percibir todas las interacciones que caracterizan su comportamiento. Identifica las diversas funciones que se llevan a cabo (producción, comercialización, contabilidad, investigación y desarrollo, etc.) como actividades de un sistema; y considera a los trabajadores, materiales, dinero, pedidos, equipos e información como entidades del mismo. La dinámica industrial estudia las características de la retroalimentación de la información en la actividad industrial con el fin de demostrar cómo la estructura, las demoras y las amplificaciones interactúan e influyen en el éxito o fracaso de la empresa.

Los pasos en un estudio de dinámica industrial son los siguientes: identificación del problema y de los objetivos a alcanzar, descripción del sistema, formulación del modelo matemático, simulación, identificación de cambios en el sistema, capacitación e implementación de las nuevas políticas.

El trabajo "The effect of environmental parameters on product recovery" [8] sirvió de base en la elección del tema de investigación. Sus autores, Georgiadis y Vlachos proponen también, un modelo de una cadena de abastecimiento cerrada. Este trabajo incluye severas modificaciones con respecto al citado. En primer lugar, el objeto de estudio es diferente. Como ya se dijo, el objetivo del presente trabajo es el análisis de la influencia del canal reverso sobre el canal tradicional mientras que el objetivo principal de Georgiadis y Vlachos es el estudio de la influencia del canal reverso sobre la demanda.

Por otra parte, el modelo desarrollado incluye muchas diferencias. Las más relevantes son: la demanda es una variable externa (no se ve afectada por la dinámica del sistema), en el canal tradicional se modela el flujo de materiales (desde la fábrica hacia los clientes) y se modela también un flujo de pedidos (desde los clientes hacia la fábrica), y por último, las demoras se modelaron constantes y de primer orden.

Para resolver el sistema de ecuaciones diferenciales que constituye el modelo se utilizó el software Mathematica, una herramienta muy poderosa a la hora de construir modelos y realizar simulaciones.

\section{Descripción del Modelo}

El sistema a estudiar es una cadena de abastecimiento cerrada constituida por un canal tradicional y un canal reverso.

La fábrica produce un único ítem que es vendido a un distribuidor. El distribuidor recibe los pedidos de los clientes y luego entrega el producto (canal tradicional). Una vez que el producto cumple su vida útil es recolectado, inspeccionado y re manufacturado. Finalmente el producto re manufacturado ingresa al inventario de productos terminados en fábrica, constituyéndose así un bucle cerrado (canal reverso). 


\subsection{Cursograma}

Un cursograma es una representación gráfica de un procedimiento [1]. Para la confección de los mismos se utilizan símbolos y reglas convencionales. Un cursograma detallado complementa a un conjunto de ecuaciones y ayuda a clarificar el modelo.

Un modelo de dinámica industrial contiene variables de nivel y variables de tasa.

Las variables de nivel son las acumulaciones a lo largo del sistema. Por ejemplo: el nivel de inventarios, el nivel de pedidos pendientes, etc. Las tasas definen los flujos entre los niveles y son resultantes de un proceso de toma de decisión.

El cursograma del modelo propuesto se nuestra en la Fig.1. Los rectángulos representan los niveles. Las válvulas representan las tasas. Las tasas pueden depender de niveles, de otras tasas o de constantes (representadas por círculos). Las líneas rosas representan el flujo de información.

La parte superior de la figura corresponde al canal tradicional. En el canal tradicional podemos distinguir dos flujos, el de materiales (líneas sin relleno) y el de pedidos (líneas rellenas). A través del flujo de materiales transitan los productos desde la fábrica al consumidor. Se observan tres variables de nivel: nivel de materias primas (Lrm: level of raw material), nivel de inventarios en fábrica (Li: level of inventory) y nivel de inventarios del distribuidor (Ldi: level of distributor). Este flujo está controlado por pedidos que se desplazan del consumidor a la fábrica.

En el flujo de pedidos se distinguen tres variables de nivel: órdenes pendientes de fabricación, pedidos del distribuidor no satisfechos y pedidos de los clientes no satisfechos.

La parte inferior del gráfico corresponde al canal reverso. Los productos usados son recolectados por la empresa o bien dispuestos por el consumidor. Los productos recolectados son inspeccionados. Los que superan dicha inspección son remanufacturados o bien dispuestos controladamente (sujeto a la capacidad de remanufactura). Finalmente, los productos remanufacturados ingresan al inventario en fábrica de productos terminados.

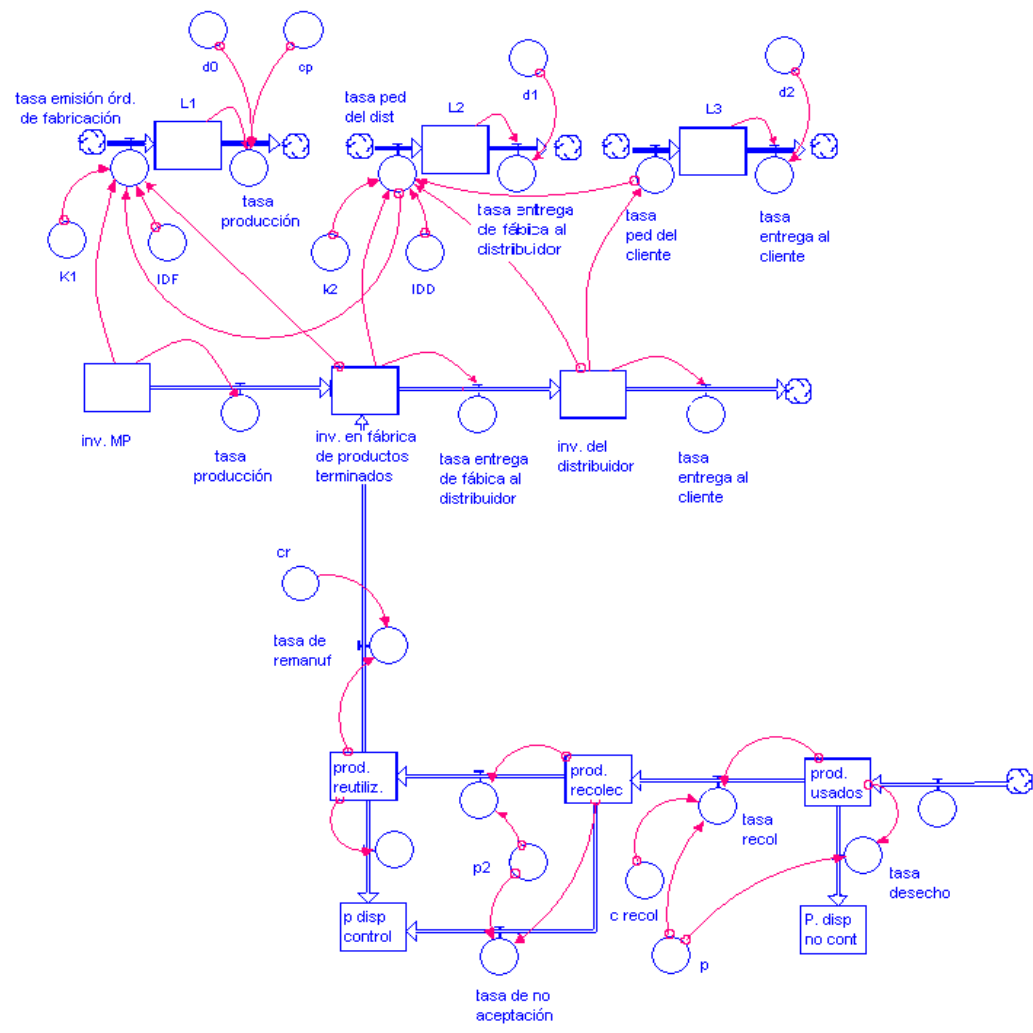

Fig. 1. Cursograma del modelo de una cadena de abastecimiento cerrada. 


\section{Formulación Matemática}

\subsection{Ecuaciones del Canal Tradicional}

Ldi(t) es el nivel de inventarios del distribuidor y queda definido por:

$$
\frac{d L d i}{d t}=R_{1}-R_{2}
$$

donde $\mathrm{R}_{1}(\mathrm{t})$ es la tasa de entrega de fábrica al distribuidor y $\mathrm{R}_{2}(\mathrm{t})$ es la tasa de entrega del distribuidor a los clientes.

$$
R_{1}(t)=\left\{\begin{array}{rl}
0, & L i(t) \leq 0 \\
\frac{L 2(t)}{d_{1}}, & L i(t) \geq 0
\end{array} .\right.
$$

donde $\operatorname{Li}(\mathrm{t})$ es el nivel de inventarios en fábrica, L2(t) es el nivel de pedidos pendientes de la fábrica al distribuidor $\mathrm{y}_{1}$ es la demora o el tiempo promedio de entrega de fábrica al distribuidor. En este trabajo se utilizan demoras de 1er orden. $\mathrm{R}_{1}(\mathrm{t})$ va a ser cero si no hay inventarios en fábrica o si no hay pedidos pendientes del distribuidor.

$$
R_{2}(t)=\left\{\begin{array}{rl}
0, & \operatorname{Ldi}(t) \leq 0 \\
\frac{L 3(t)}{d_{2}}, & \operatorname{Ldi}(t) \geq 0
\end{array} .\right.
$$

donde L3(t) es el nivel de pedidos pendientes de los clientes y $\mathrm{d}_{2}$ es el tiempo promedio de entrega del distribuidor al cliente.

$\mathrm{Li}(\mathrm{t})$, como ya se dijo, es el nivel de inventarios en fábrica y queda definido por:

$$
\frac{d L i}{d t}=R_{p}+T_{2}-R_{1}
$$

donde $\mathrm{Rp}(\mathrm{t})$ es la tasa de producción, $\mathrm{R}_{1}(\mathrm{t})$ como ya se explicó, es la tasa de entrega de fábrica al distribuidor y $\mathrm{T}_{2}(\mathrm{t})$ es la tasa de remanufactura la cual se define en el canal reverso.

$$
R_{P}(t)=\left\{\begin{aligned}
0, & \operatorname{Lrm}(t) \leq 0 \\
\operatorname{Min}\left\{\frac{L 1(t)}{d_{0}} ; c_{p}\right\}, & \operatorname{Lrm}(t) \geq 0
\end{aligned}\right.
$$


donde $\mathrm{d}_{0}$ es una demora del sistema (tiempo promedio de producción), $\mathrm{c}_{\mathrm{p}}$ es la capacidad de producción, $\operatorname{Lrm}(\mathrm{t})$ es el nivel de materias primas y L1(t) es el nivel de órdenes de fabricación pendientes. La tasa de producción va hacer nula en los casos en que no haya materia prima o que no haya órdenes de fabricación pendientes. La tasa de fabricación nunca puede superar la capacidad de producción.

$\operatorname{Lrm}(\mathrm{t})$ es el nivel de materias primas y está definido por:

$$
\frac{d L r m}{d t}=-R_{p}
$$

ya que el modelo no incluye el reaprovisionamiento de materia prima.

Todas las tasas descritas hasta aquí son tasas de entrega de mercadería. La tasa de producción, $\mathrm{Rp}(\mathrm{t})$ representa la entrega de productos dentro de la misma fábrica desde el sector de producción a los almacenes de productos terminados. $\mathrm{R}_{1}(\mathrm{t})$ es la tasa de entrega de la fábrica al distribuidor y $\mathrm{R}_{2}(\mathrm{t})$ es la tasa de entrega al cliente.

Las ecuaciones que definen las variables de nivel del flujo de pedidos son las siguientes:

L3(t) es el nivel de pedidos pendientes de los clientes y está definido por:

$$
\frac{d L 3}{d t}=R_{4}-R_{2}
$$

donde $\mathrm{R}_{2}(\mathrm{t})$ es la tasa a la cual el distribuidor entrega los productos a los clientes y $\mathrm{R}_{4}(\mathrm{t})$ es la tasa de pedidos de los clientes aceptados por la empresa, se define de la siguiente forma:

$$
R_{4}(t)=\left\{\begin{array}{rl}
0, & L d i(t) \leq 0 \\
d(t), & L d i(t) \geq 0
\end{array} .\right.
$$

O sea, si el distribuidor no tiene inventarios, no se toman pedidos de los clientes. Esta política de decisión se fijo en base a que el modelo no considera el reaprovisionamiento de materia prima Ec. (6). d(t) es la demanda, variable externa del modelo.

L2(t) es el nivel de pedidos pendientes de la fábrica al distribuidor. Queda definido por:

$$
\frac{d L 2}{d t}=R_{3}-R_{1}
$$

donde $R_{1}(t)$ es la tasa a la cual la fábrica entrega los productos al distribuidor y $\mathrm{R}_{3}(\mathrm{t})$ es la tasa a la cual el distribuidor pide a la fábrica. $\mathrm{R}_{3}(\mathrm{t})$ está definida por,

$$
R_{3}(t)=\left\{\begin{array}{r}
0, \quad L i(t) \leq 0 \\
R_{4}(t)+k_{2} \cdot(I D D-L d i(t)), \quad L i(t) \geq 0
\end{array} .\right.
$$


donde $\mathrm{k}_{2}$ es una constante de pedidos e IDD es el nivel de inventarios deseado por el distribuidor. Esta tasa de pedidos es una decisión del distribuidor. El solo igualar la tasa de pedidos a la demanda no es satisfactorio. Cuando la demanda aumenta, el inventario se agota debido a que la tasa de entregas requiere cierto tiempo para ponerse al corriente con la nueva tasa de demanda. Análogamente, una caída en la tasa de demanda hace que se eleve el nivel de inventarios. Por lo tanto se decide agregar a la tasa de demanda un término proporcional a la diferencia entre los niveles deseado y real. Cuando el inventario está bajo, esto aumenta la tasa de pedidos y cuando el inventario es alto, disminuye los pedidos. Esta política de pedidos introduce la retroalimentación y condiciona los resultados. El valor de $\mathrm{k}_{2}$ afectará la respuesta del sistema, ya que $\mathrm{k}_{2}$ corresponde a la amplificación en el ciclo de retroalimentación. Cuando la fábrica se queda sin inventarios no se aceptan pedidos del distribuidor.

L1(t) es el nivel de órdenes de fabricación pendientes y está definido por:

$$
\frac{d L 1}{d t}=R_{0}-R_{p}
$$

donde $\mathrm{Rp}(\mathrm{t})$ es la tasa de producción y $\mathrm{R}_{0}(\mathrm{t})$ es la tasa a la cual se generan las órdenes de fabricación y queda definida por,

$$
R_{0}(t)=\left\{\begin{array}{r}
0, \quad \operatorname{Lrm}(t) \leq 0 \\
R_{3}(t)+k_{1} \cdot(I D F-\operatorname{Li}(t)), \operatorname{Lrm}(t) \geq 0
\end{array} .\right.
$$

donde $\mathrm{k}_{1}$ es otra constante de pedido e IDF es el nivel deseado de inventario en fábrica. $\mathrm{R}_{0}(\mathrm{t})$ es la tasa a la cual la fabrica emite las ordenes de fabricación por lo tanto su interpretación es similar a la de una tasa de pedidos, en este caso pedidos de fabricación emitidos por la misma fábrica. No se pueden emitir órdenes de fabricación si la materia prima se ha agotado.

Las nuevas tasas definidas en el flujo de pedidos son tasas de pedidos.

Por lo tanto, se pueden distinguir dos tipos de ecuaciones de tasas. Las tasas de entrega que dependen del nivel de pedidos pendientes, de la demora promedio que se produce en entregar el producto en cada nivel y de la existencia de inventarios a partir de los cuales cumplir con la entrega.

Las tasas de pedidos representan decisiones en función de los niveles de inventarios reales, de los niveles de inventarios deseados, de los pedidos recibidos y de la existencia de mercaderías en el eslabón anterior (al que se le hace el pedido). Estas políticas de reposición condicionan los resultados de la simulación.

\subsection{Ecuaciones del Canal Reverso}

El canal reverso queda definido por cinco ecuaciones de nivel y las respectivas ecuaciones de tasa.

$\mathrm{Pu}(\mathrm{t})$ es el nivel de productos usados. Queda definido por:

$$
\frac{d P u}{d t}=T-T_{1}-T_{4}
$$


donde $\mathrm{T}(\mathrm{t})$ es la tasa a la cual los productos finalizan su vida útil (salen del mercado), $\mathrm{T}_{1}(\mathrm{t})$ es la tasa de recolección de la empresa y $\mathrm{T}_{4}(\mathrm{t})$ es la tasa de desecho.

$$
T(t)=R_{2}(t) .
$$

Se supone que la tasa a la cual los productos salen del mercado (terminan su vida útil) es igual a la tasa a la cual ingresan al mercado (o sea es igual a la tasa de entrega al cliente). O sea, se considera que se compra un nuevo producto cada vez que otro finaliza su vida útil.

$$
T_{1}=\operatorname{Min}\left\{p \cdot P u(t), c_{r e c}\right\} .
$$

donde $\mathrm{p}$ es el porcentaje de recolección y $\mathrm{c}_{\mathrm{rec}}$ es la capacidad de recolección, consecuencia de la decisión de la administración. $T_{1}(t)$ no puede ser mayor a $c_{\text {rec }}$.

$$
T_{4}(t)=\left\{\begin{array}{rl}
(1-p) \cdot P u(t), & p \cdot P u(t) \leq c_{r e c} \\
P u(t)-c_{r e c}, & p \cdot P u(t) \geq c_{r e c}
\end{array} .\right.
$$

Los clientes desechan los productos que no son recolectados por la empresa.

Prec(t) es el nivel de productos recolectados. Está dado por:

$$
\frac{d \text { Prec }}{d t}=T_{1}-T_{a}-T_{n a} .
$$

donde $T_{1}(t)$ es la tasa de recolección, $T_{a}(t)$ es la tasa de aceptación del proceso de inspección y $\mathrm{T}_{\mathrm{na}}(\mathrm{t})$ es la tasa de rechazo.

$$
T_{a}(t)=p_{2} \cdot \operatorname{Prec}(t) .
$$

donde $\mathrm{p}_{2}$ es el porcentaje de aceptación promedio del proceso de inspección.

$$
T_{n a}(t)=\left(1-p_{2}\right) \cdot \operatorname{Prec}(t) .
$$

Preut(t) es el nivel de productos reutilizables o sea que pueden llegar a ser remanufacturados. Queda definido por:

$$
\frac{d \text { Preut }}{d t}=T_{a}-T_{2}-T_{3} .
$$

donde $T_{a}(t)$ es la tasa de aceptación del proceso de inspección, $T_{2}(t)$ es la tasa de remanufactura y $\mathrm{T}_{3}(\mathrm{t})$ es la tasa a la cual todo producto reutilizable, no re manufacturado es dispuesto controladamente por la empresa.

$$
T_{2}(t)=\operatorname{Min}\left\{c_{r}, \operatorname{Preut}(t)\right\} .
$$

donde $c_{r}$ es la capacidad actual de remanufactura. $T_{2}(t)$ nunca puede ser mayor a $c_{r}$. 


$$
T_{3}(t)=\operatorname{Max}\left\{\operatorname{Preut}(t)-c_{r}, 0\right\} .
$$

No se modelan las posibles demoras en las actividades de recolección, inspección y remanufactura ya que se suponen muy pequeñas frente a las demoras incluidas en el canal tradicional.

\section{Resolución del Sistema de Ecuaciones}

El sistema de ecuaciones diferenciales $[4,11,12]$ Ec. $(1,4,6,7,9,11,13,17,20)$ junto a las ecuaciones de tasa Ec. $(2,3,5,8,10,12,14,15,16,18,19,21,22)$ constituyen el modelo, el mismo se resolvió numéricamente [2] utilizando el utilitario Mathematica.

El sistema de ecuaciones resultante es rígido. De esta forma se usaron pasos de integración más grandes cuando el sistema no presentaba rigidez (dinámica lenta) y pasos más pequeños cuando si la presentaba, evitando así un excesivo trabajo computacional.

\section{Parámetros del Modelo}

Los inventarios deseados en fábrica y en el distribuidor se fijaron en $0,5 \mathrm{t}$ y $1 \mathrm{t}$ respectivamente. La demora promedio entre que se genera una orden de producción y se almacena el producto en fábrica, se fijó en 2 días. Entre que se genera un pedido a fábrica y se entrega el producto al distribuidor, la demora se fijó en 3 días. Finalmente, la demora entre que un cliente pide un producto y el distribuidor se lo entrega se fijó en 5 días.

Para simplificar nuestro análisis consideramos la capacidad de producción, de recolección y de remanufactura lo suficientemente grandes tal que no sean un factor limitante.

Las constantes de pedidos, $\mathrm{k}_{1} \mathrm{y}_{2}$, fueron fijadas ambas en 0,5 . El porcentaje de recolección $\mathrm{p}$, fue fijado en $40 \%$ y el porcentaje de productos que superan la inspección $\mathrm{p}_{2}$, en $85 \%$.

\section{Condiciones Iniciales}

Los valores iniciales de las variables se eligieron de forma tal que el sistema comience a partir de una solución estacionaria. Cualquier perturbación al sistema provocará un alejamiento de las condiciones iniciales.

El nivel de materias primas se inicializó en 150 t. Los niveles de inventarios en fábrica y en el distribuidor se inicializaron en sus respectivos niveles deseados de $0,5 \mathrm{t}$ y 1 t. Las órdenes de fabricación pendientes en 3 t, los pedidos pendientes del distribuidor en 4,5 t y los del cliente en 7,5 t. Con respecto al canal reverso, todos sus niveles se inicializaron en cero. 


\section{Resultados de la Simulación}

Las corridas de simulación se realizaron para dos casos diferentes de demanda. El primero, de demanda constante e igual a 1,5 t por día hasta el día 20 y luego se introdujo un incremento de $1 \mathrm{t}$ por día (Fig. 7.1.). Finalmente se corrió el modelo para una demanda estacional con una media de 1.5 t por día (Fig. 7.2.).

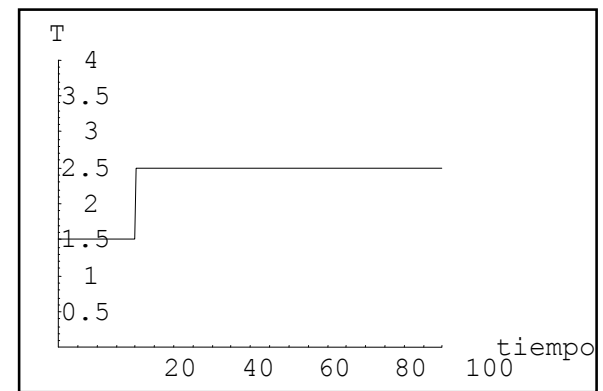

Fig. 7.1. Demanda con escalón.

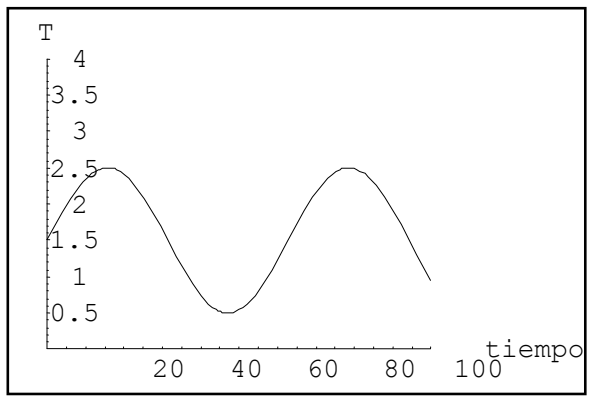

Fig. 7.2. Demanda estacional.

En cada caso, se realizó primero la simulación del canal tradicional o sea, del modelo sin recolección. Luego se simuló el modelo completo (canal tradicional y canal reverso), con el objetivo de analizar los cambios introducidos por el canal reverso en el comportamiento de las variables del canal tradicional.

A continuación se presentan los resultados obtenidos.

\subsection{Análisis del Tiempo de Agotamiento de la Materia Prima}

Para llevar a cabo este análisis se corrió el modelo completo (canal tradicional y canal reverso) con diferentes valores de porcentaje de recolección (p).

\subsubsection{Demanda con Escalón}

La Fig. 7.3. contiene los resultados obtenidos en las diferentes corridas para distintos valores de $\mathrm{p}(\mathrm{p}=0, \mathrm{p}=0,2, \mathrm{p}=0,4, \mathrm{p}=0,6, \mathrm{p}=0,8 \mathrm{y} \mathrm{p}=1)$. Como era de esperar, el tiempo de agotamiento de la materia prima es mayor cuanto mayor es el porcentaje de recolección. 


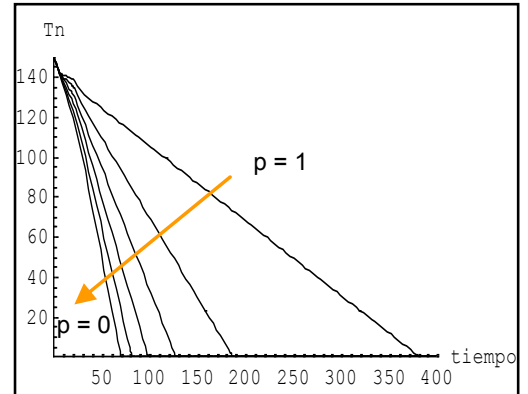

Fig. 7.3. Tiempo de agotamiento de la MP en función del porcentaje de recolección

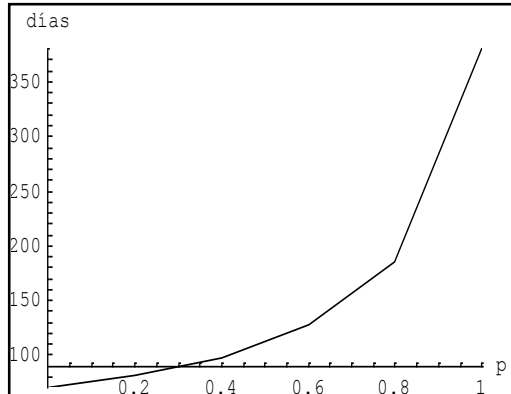

Fig. 7.4. Tiempo de agotamiento de la MP en función del porcentaje de recolección

La Fig. 7.4. relaciona el porcentaje de recolección con los tiempos de agotamiento. Se observa que el aumento en la duración de la materia prima no es proporcional al aumento en el porcentaje de recolección.

\subsubsection{Demanda Estacional}

La Fig. 7.5. contiene las curvas obtenidas en las diferentes corridas. Se observa el mismo comportamiento que en el caso anterior: a mayor porcentaje de recolección, mayor tiempo de duración de la materia prima.

En la Fig. 7.6. se observa que, en este caso la relación entre p y el tiempo de agotamiento tampoco es proporcional. Más aún, ambos casos de demanda analizados presentan curvas similares.

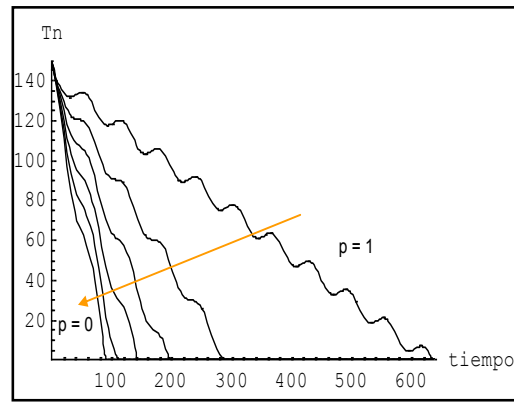

Fig. 7.5. Tiempo de agotamiento de la MP en función del porcentaje de recolección

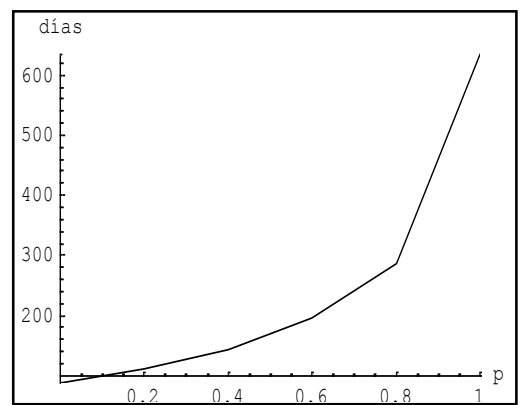

Fig. 7.6. Tiempo de agotamiento de la MP en función del porcentaje de recolección

\subsubsection{Conclusión}

Además de confirmar la conclusión intuitiva de que con la recolección se incremente el tiempo de agotamiento de la materia prima, el obtener la curva que relaciona ambas variables nos brinda mayor información al respecto. En los dos casos analizados, las 
curvas obtenidas son similares. Se ve que la relación no es proporcional. Para porcentajes de recolección menores los cambios que se producen en el tiempo de agotamiento de la materia prima son más suaves que para porcentajes mayores.

Cabe aclarar, que para todas las corridas se utilizó un porcentaje de aceptación del proceso de inspección $\left(\mathrm{p}_{2}\right)$ de $85 \%$. Razón por la cual la materia prima también se agota con $\mathrm{p}=1$.

\subsection{Análisis del Comportamiento de los Inventarios}

Para realizar este análisis se realizaron corridas de ambos modelos (sin recolección y con recolección) para distintos valores de las constantes de pedidos $\mathrm{k}_{1} \mathrm{y}_{2}$.

Cabe recordar, que la política de generación de órdenes de fabricación se definió de la siguiente manera:

$$
R_{0}(t)=\left\{\begin{array}{r}
0, \operatorname{Lrm}(t) \leq 0 \\
R_{3}(t)+k_{1} \cdot(I D F-\operatorname{Li}(t)), \operatorname{Lrm}(t) \geq 0
\end{array} .\right.
$$

y la política de pedidos del distribuidor se definió como:

$$
R_{3}(t)=\left\{\begin{array}{r}
0, L i(t) \leq 0 \\
R_{4}(t)+k_{2} \cdot(I D D-L d i(t)), L i(t) \geq 0
\end{array} .\right.
$$

donde $\mathrm{R}_{4}(\mathrm{t})$ es la tasa de pedidos de los clientes aceptados por la empresa.

Si no hay materia prima no se generan órdenes de fabricación. Si no hay inventario de producto terminado en fábrica, el distribuidor no genera pedidos. Esto se definió así porque el modelo no considera el reabastecimiento de materia prima.

Ambas políticas incluyen un término proporcional a la diferencia entre el inventario deseado y el real. Por lo tanto cuando el inventario está bajo, esto aumenta la tasa de pedidos y cuando el inventario es alto, disminuye los pedidos. Estas políticas de pedidos introducen la retroalimentación. El valor de $\mathrm{k}_{1} \mathrm{y}$ de $\mathrm{k}_{2}$ afectará la respuesta del sistema, ya que corresponden a la amplificación en el ciclo de retroalimentación.

A continuación se muestran los resultados obtenidos para el caso de la demanda con escalón. Los resultados obtenidos con demanda estacional son similares.

\subsubsection{Demanda con Escalón. Canal Tradicional}

En la Fig. 7.7. y en la Fig. 7.8. se ve que se producen oscilaciones en el sistema. Sin embargo la política de pedidos adoptada logra su objetivo ya que los inventarios se estabilizan en el nivel planificado.

En la Fig. 7.7. se observa que las oscilaciones en el inventario de fábrica son de mayor frecuencia cuando $\mathrm{k}_{1}=1 \mathrm{y}$ de mayor amplitud cuando $\mathrm{k}_{1}$ decrece. Esto es así ya que por ejemplo, cuando $k_{1}=1$ la fábrica genera tantas órdenes de fabricación como para cubrir el $100 \%$ del déficit entre el inventario real y el deseado, pero como 
hay una demora de dos días $\left(\mathrm{d}_{0}\right)$ en la fabricación, se pide nuevamente para cubrir el déficit.

El mismo comportamiento se ve en la Fig. 7.8. para el inventario del distribuidor.

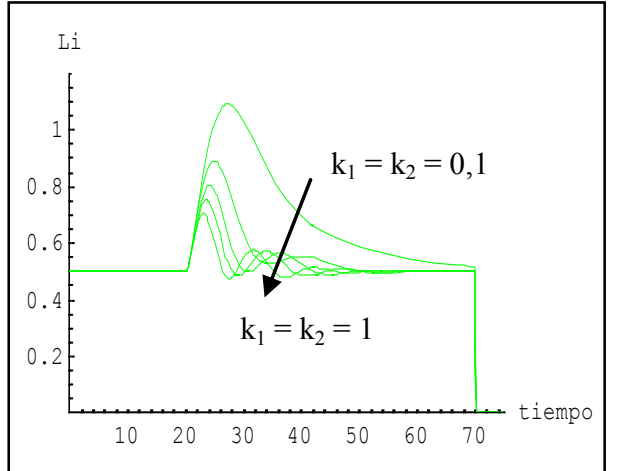

Fig. 7.7. Comportamiento del inventario en fábrica ante diferentes valores de $\mathrm{k} 1 \mathrm{y} \mathrm{k} 2$. Modelo sin recolección

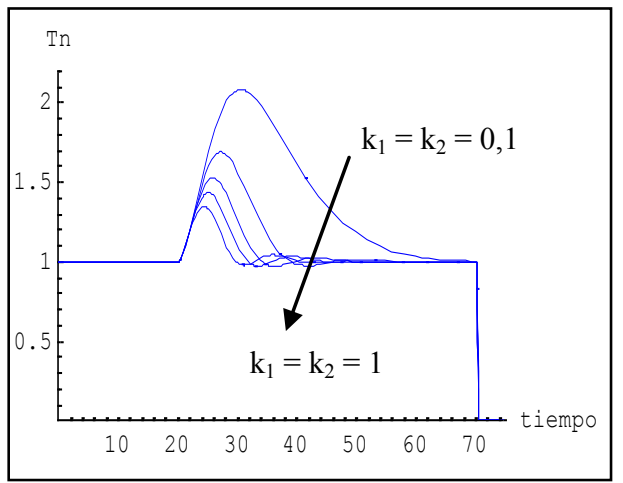

Fig. 7.8. Comportamiento del inventario del distribuidor ante diferentes valores de $\mathrm{k}_{1} \mathrm{y}_{2}$. Modelo sin recolección

\subsubsection{Demanda con Escalón. Modelo Completo: Canal Tradicional y Canal Reverso}

En este caso se observa el mismo fenómeno que en el caso anterior. A mayor constante de pedido, mayor frecuencia de oscilaciones de menor amplitud.

En el inventario de fábrica (Fig. 7.9.) se suma además otro fenómeno. Desde $t=0$, el inventario comienza a incrementarse desde su nivel deseado tendiendo a estabilizarse en un nivel superior al planificado. Esto ocurre por el flujo de productos re manufacturados que llegan al inventario de fábrica a través del canal reverso. Luego en $\mathrm{t}=20$, se produce el incremento en la demanda, y los inventarios comienzan a elevarse nuevamente estabilizándose en un nivel aún superior. Este efecto es más notable con $\mathrm{k}_{1}$ pequeño ya que en este caso, en la generación de órdenes se da poco peso a la diferencia entre el nivel deseado y el real. En la Fig. 7.9. se puede ver también la ampliación de la zona marcada para apreciar mejor las oscilaciones presentes en el sistema.

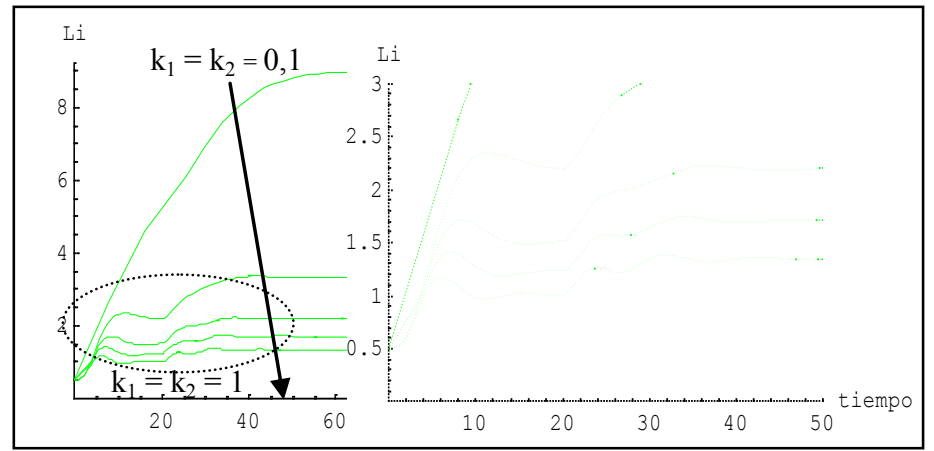

Fig. 7.9. Comportamiento del inventario en fábrica ante diferentes valores de $\mathrm{k}_{1} \mathrm{y} \mathrm{k}_{2}$. Modelo con recolección 
En el comportamiento del inventario del distribuidor no se observan cambios. Esto es así ya que el flujo proveniente del canal reverso influye directamente sobre el nivel de inventarios en fábrica, lo cual no produce ningún efecto sobre la política de pedidos del distribuidor.

\subsubsection{Modificación de la Política de Generación de Ordenes de Fabricación}

Al incluir en el modelo el canal reverso, no se modificó la política de generación de órdenes de fabricación (Ec.12) produciéndose así un incremento en los stocks de fábrica (Fig. 7.9.).

A continuación se modifica la política de pedido (generación de órdenes de fabricación) de manera tal que considere el flujo de productos re manufacturados.

$$
R_{0}^{\prime}(t)=\left\{\begin{array}{r}
0, \operatorname{Lrm}(t) \leq 0 \\
R_{3}(t)+k_{1} .(I D F-\operatorname{Li}(t))-\boldsymbol{T}_{2}(\boldsymbol{t}), \operatorname{Lrm}(t) \geq 0
\end{array} .\right.
$$

donde $\mathrm{T}_{2}(\mathrm{t})$ es la tasa de re manufactura.

El comportamiento del inventario de fábrica se ha modificado (Fig. 7.11.). El inventario comienza a incrementar su nivel, alejándose del nivel deseado de $0,5 \mathrm{t}$, oscila y tiende a estabilizarse en $0,5 \mathrm{t}$ cuando en $\mathrm{t}=20$ se produce el incremento en la demanda, produciendo una nueva oscilación. Finalmente la nueva política adoptada logra su objetivo y el inventario se estabiliza en el nivel planeado.

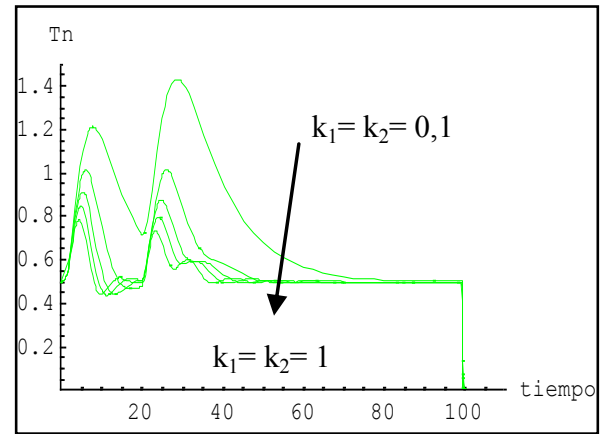

Fig. 7.11. Comportamiento del inventario en fábrica ante diferentes valores de $\mathrm{k}_{1} \mathrm{y} \mathrm{k}_{2}$. Modelo con recolección.

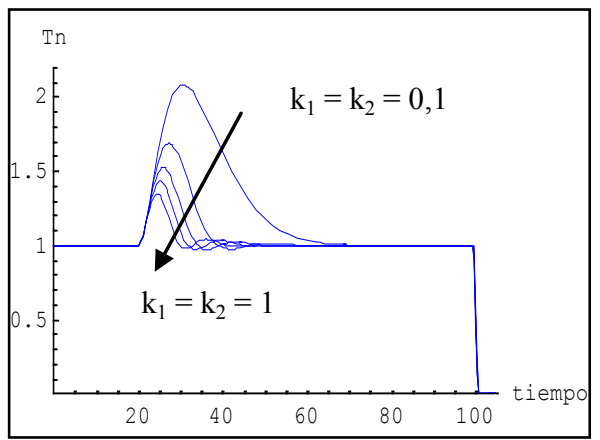

Fig.7.12.Comportamiento del inventario del distribuidor ante diferentes valores de $\mathrm{k}_{1} \mathrm{y}_{2}$. Modelo con recolección..

Como era de esperar, el inventario del distribuidor no sufrió modificaciones ya que solo se modificó la política de generación de órdenes de fabricación (Fig. 7.12.). 


\section{Conclusión}

El modelo propuesto es una herramienta experimental, que con la correcta selección de parámetros puede ayudar a analizar diferentes escenarios y a responder preguntas sobre el comportamiento de cadenas de abastecimientos cerradas.

En este caso el análisis se centró en el comportamiento de los inventarios. Más precisamente en el efecto que produce el canal reverso sobre los mismos. En cuanto al inventario de materias primas, se analizó la forma en que el porcentaje de recolección afecta el tiempo de agotamiento de las mismas, obteniendo la curva que relaciona ambas variables. Con respecto al inventario en fábrica de productos terminados, se observó como el flujo de productos re manufacturados hace que estos se estabilicen en un nivel superior al deseado. También se encontró que este efecto adverso es más pronunciado cuando se utilizan constantes de pedidos $\left(\mathrm{k}_{1}\right)$ pequeñas. Para revertir esta situación se propuso la modificación de la política de generación de órdenes de producción. En cuanto a los inventarios del distribuidor, se advirtió que la inclusión del canal reverso no produce cambios en su comportamiento. Se analizó también, la influencia de la constante de pedido $\mathrm{k}_{2}$ sobre los mismos.

Es importante destacar la utilidad del modelo como herramienta experimental ya que hubiese sido casi imposible realizar dichos análisis sobre un sistema real.

El modelo presentado se desarrolló en forma general para facilitar su aplicación a diferentes casos prácticos. Realizando cambios en el mismo y en los valores adoptados por los parámetros, este se puede adaptar para estudiar diferentes casos.

Por ejemplo, se podría incluir el reaprovisionamiento de materia prima cuando el nivel de las mismas llega a un valor predefinido, y analizar cómo influye y como sería en este caso la dinámica del sistema.

Otra opción sería adaptar el modelo tal que el producto recuperado se utilice nuevamente como materia prima del proceso productivo. De esta forma el modelo se podría aplicar al reciclado de latas de aluminio, baterías de plomo, papel, etc.

También se podría modelar la influencia que pueden tener las actividades de recolección, remanufactura o reciclado sobre la demanda de ciertos productos.

Todas estas diferentes posibilidades se pueden combinar dando lugar a nuevas opciones.

En todos los casos, un tópico interesante para futuras investigaciones es asociar costos a cada operación específica y buscar políticas óptimas. 


\section{Referencias}

1. Betria, Ma. de Lujan et al: Dinámica Industrial Aplicada a la Logística Reversa. Proyecto Final de Ingeniería Industrial, FCEIA, UNR (2006)

2. Canale, Raymond P., Chapra, Steven C.: Métodos numéricos para ingenieros $2^{\mathrm{da}} \mathrm{ed}$. Mc Graw Hill, México (1994)

3. Cover, J. M.: Introducción a la Dinámica de Sistemas. Cacit Group (1999)

4. Edwars, C. H. Jr y Penny, David E.: Ecuaciones Diferenciales Elementales y Problemas con Condiciones en la Frontera $3^{\mathrm{a}}$ ed. Prentice Hall, México (1994)

5. Fleischmann, Moritz, et al: Quantitative models for reverse logistics: A review. European Journal of Operational Research. 103, 1--17 (1997)

6. Forrester, Jay W.: Dinámica Industrial $2^{\mathrm{a}}$ ed. El Ateneo, Buenos Aires (1981)

7. Forrester, Jay W.: System Dynamics, Systems Thinfing, and Sof OR. System Dynamics Review. 10 (1994)

8. Georgiadis P., Vlachos D.: The effect of environmental parameters on product recovery. European Journal of Operational Research. 157, 449--464 (2004)

9. Gordon, Geoffrey: Simulación de Sistemas. Diana S.A., México (1981)

10. Leite, R. P.: Logística reversa: nueva área de logística empresarial. Tecnologística. 78 (2002)

11. Otto Plaat: Ecuaciones Diferenciales Ordinarias. Reverté S.A., Barcelona (1974)

12. Simmons, George F.: Ecuaciones diferenciales con aplicaciones y notas históricas $2^{\mathrm{a}}$ ed. Mc Graw Hill, España (1993) 